Original Article

\title{
Morphological changes of lower leg muscles according to ankle joint position during sitting evaluated by gravity mri in young females
}

\author{
Ryo Miyachi, PT, PhD ${ }^{1,2)}$, Toshiaki Yamazaki, PT, PhD ${ }^{2 *}$, NaOki Ohno, RT, $\mathrm{PhD}^{2)}$, \\ Tosiaki Miyati, $\left.\mathrm{PhD}, \mathrm{dmSc}^{2}\right)$ \\ 1) Department of Rehabilitation, Ishikawa-ken Saiseikai Kanazawa Hospital, Japan \\ 2) Faculty of Health Sciences, Institute of Medical, Pharmaceutical and Health Sciences, Kanazawa \\ University: 5-11-80 Kodatsuno, Kanazawa, Ishikawa 920-0942, Japan
}

\begin{abstract}
Purpose] This study aimed to clarify whether the morphological changes of the lower leg muscle occur equally in the longitudinal direction of the muscle according to changes in ankle joint position during sitting. [Participants and Methods] The participants were 15 healthy young females whose dominant lower legs were analyzed. The participants sat with the lower leg vertical to the floor with a neutral, dorsiflexed, or plantarflexed ankle position. Images were obtained from the fibular head from $290 \mathrm{~mm}$ distal using gravity magnetic resonance imaging. The muscle cross-sectional areas of the soleus, medial and lateral heads of the gastrocnemius, and anterior tibialis were measured. [Results] The muscle cross-sectional area of the soleus at the $1 / 4$ proximal muscle belly in the plantarflexed position was greater than those in the other positions. The $1 / 4$ distal part in the plantarflexed position was smaller than those in the other positions. The muscle cross-sectional area of the gastrocnemius at the $1 / 4$ distal part in the plantarflexed position was smaller than that in the dorsiflexed position. In contrast, the muscle cross-sectional area of the tibialis anterior at the $1 / 4$ proximal part in the dorsiflexed position was greater than those in the other positions, while that at the $1 / 4$ distal part in the dorsiflexed position was smaller than that in the plantarflexed position. [Conclusion] In the sitting position, the morphological changes of the lower leg muscle according to changes in ankle joint position are not uniform in the longitudinal muscle direction. Key words: Gravity MRI, Longitudinal morphological change, Lower leg muscle
\end{abstract}

(This article was submitted Dec. 29, 2018, and was accepted Mar. 2, 2019)

\section{INTRODUCTION}

The lower leg muscles play many roles beyond simple movement of the ankle, such as assisting in posture $\operatorname{control}^{1,2)}$ and gait $^{3,4)}$. Decline in the strength of the lower leg muscles due to limited exercise or reduced activity needs to be prevented. In order to prevent inactivity such as after surgery, inpatients are often advised to assume a sitting position that can hold easily with little risk of falling during the day. In addition, stretching a muscle increases its length and inhibits disuse muscle atrophy ${ }^{5}$. Passive positioning of the legs' position can be performed without assistance. Thus, using times when a patient is sitting during the day to adjust the position of the ankle is an effective use of time outside of physical therapy sessions, and can maintain the strength of the lower leg muscles.

Few previous studies on stretching of the lower leg muscles while in a sitting position have reported information on the morphology of the lower leg muscles. One study reported that inhibition of muscle atrophy as a result of stretching differs along the longitudinal axis of a muscle ${ }^{5}$. The load due to stretching may differ along the longitudinal axis of a muscle.

*Corresponding author. Toshiaki Yamazaki (E-mail: yamazaki@mhs.mp.kanazawa-u.ac.jp)

(C2019 The Society of Physical Therapy Science. Published by IPEC Inc.

(c) (1) $\odot$ This is an open-access article distributed under the terms of the Creative Commons Attribution Non-Commercial No DerivaCC BY NC ND tives (by-nc-nd) License. (CC-BY-NC-ND 4.0: https://creativecommons.org/licenses/by-nc-nd/4.0/) 
Implementing other interventions at sites that show little morphological change upon stretching may better inhibit a decline in muscle output. However, the exact locations of morphological changes resulting from a stretch stimulus under the force of gravity, together with the extents of those changes, remain unclear. Therefore, it is necessary to investigate the basic morphological information of the muscle to the stretch stimulus at the anti-gravitational position.

Ultrasonography is available as a method for obtaining morphological information about a muscle in a sitting position, but cannot provide information on the whole muscle. Magnetic resonance imaging (MRI) offers accurate 3-dimensional morphological data over wide areas, but can only be performed in limited body positions. There have been many MRI studies on the morphology of the lower leg muscles in the decubitus position ${ }^{6-8)}$, but no study in the sitting position. Recently, Gravity MRI with a 0.4 -T permanent magnet (Gravity MRI) ${ }^{9}$ was developed and put into practical use by co-researchers. Gravity MRI can be performed in any body position, allowing for the acquisition of morphological data in anti-gravity limb positions. Using this method, the lower leg muscle morphology over a wide area can be evaluated in the sitting position.

The purpose of this study was to obtain basic information on the morphological changes of lower leg muscle depending on shortening or stretching, and to clarify whether the morphological changes occur equally in the longitudinal direction of the muscle while sitting.

\section{PARTICIPANTS AND METHODS}

The participants were fifteen healthy young females with no complaints of pain affecting daily life. Mean ( \pm standard deviation) age, height, and weight were $21.0 \pm 1.0$ years, $160.4 \pm 3.8 \mathrm{~cm}$, and $52.4 \pm 7.3 \mathrm{~kg}$, respectively. None of the participants had a history of orthopedic problems of the legs or vertebrae. Considering the relationship between the length of the lower leg and the length of the imaging coil for MRI, and that the muscle belly of is more readily evident in females, participants in the current study were female.

The protocol for this study was approved by the Ethics Committee of our university (approval number: 687). Written informed consent was obtained from all participants.

Each participant on a chair with their arms crossed on their chest, knee flexion at $90^{\circ}$, and their lower legs perpendicular to the floor. The chair had a backrest and no elbow rest. Images were randomly acquired in 3 positions: the neutral position between dorsi- and plantarflexion, $20^{\circ}$ of dorsiflexion, and $20^{\circ}$ of plantarflexion. The lower limb was placed on a handmade tilt table to set the position of the ankle joint. The seat height was adjusted by putting a plate on the chair to make the knee flexion $90^{\circ}$ for each ankle position. The thighs were fixed with cushions and bands, and the participants were instructed to remain motionless as much as possible during the measurement. Using Gravity MRI (Hitachi Healthcare), horizontal T1-weighted images were obtained from the fibular head to $290 \mathrm{~mm}$ distal at $10 \mathrm{~mm}$ intervals. The imaging parameters were as follows: slice plane axial, pulse sequence RF-spoiled steady state gradient echo, field of view $280 \mathrm{~mm}$, repetition time $110.0 \mathrm{~ms}$, echo time $8.6 \mathrm{~ms}$, flip angle 35 degrees, slice thickness $10.0 \mathrm{~mm}$, slice interval $10.0 \mathrm{~mm}$, matrix size $256 \times 256$, number of signals averaged $=2$, receiver bandwidth $20.6 \mathrm{kHz}$, and scan time $4 \mathrm{~min} 32 \mathrm{~s}$.

The anatomical muscle cross-sectional areas (MCSA) of the soleus (SOL), and gastrocnemius medial head (GM), gastrocnemius lateral head (GL), anterior tibial tuberosity (TA) were measured using the Image J image analysis program. In the measurement, the ankle position was blinded in order to avoid the detection bias. All statistical analyses were carried out using SPSS ver.19 (Japan IBM). Values of $\mathrm{p}<0.05$ were considered significant. For comparison of MCSA at each position, 5 points — endmost muscle belly, most proximal and most distal, and 1/4 proximal, middle, and 1/4 distal — were extracted for each muscle, and repeated-measures analysis of variance was performed on each point. The Bonferroni multiple comparison was used for post-hoc tests. To extract the maximum MCSA value, repeated-measures analysis of variance and the Bonferroni multiple comparison were again used. All data are expressed as mean $\pm \mathrm{SD}$ (standard deviation).

\section{RESULTS}

In Table 1, the SOL MCSA of most proximal and middle muscle belly showed no significant difference at each ankle position. SOL MCSA of 1/4 proximal muscle belly was significantly greater for plantarflexion than for neutral and dorsiflexion. In contrast, SOL MCSA of distal 1/4 muscle belly was significantly greater for dorsiflexion and neutral than for plantarflexion.

For GM MCSA, the $1 / 4$ distal muscle belly was significantly greater for dorsiflexion than for neutral and plantarflexion, but not significantly different in other parts.

GL MCSA at 1/4 distal was significantly greater only for dorsiflexion and neutral compared to plantarflexion.

TA proximal, middle and most distal muscle belly MCSA values were not significantly different among ankle positions. TA MCSA at $1 / 4$ proximal muscle belly was significantly greater for dorsiflexion than for neutral and plantarflexion. In contrast, TA MCSA at 1/4 distal muscle belly was significantly greater for plantarflexion than for dorsiflexion.

Comparisons of the maximum MCSA values for each muscle (Table 2) showed no significant difference in the SOL, but in the TA they were significantly greater for neutral and dorsiflexion than for plantarflexion. In both GM and GL, the maximum MCSA was significantly greater for neutral and plantarflexion than for dorsiflexion. 
Table 1. Comparison of the muscle cross-sectional area (MCSA) values for each muscle at different ankle joint positions

\begin{tabular}{|c|c|c|c|c|c|c|c|c|}
\hline & $\begin{array}{l}\text { Ankle joint } \\
\text { position }\end{array}$ & $\begin{array}{l}\text { MCSA of most } \\
\text { proximal region } \\
\left(\mathrm{cm}^{2}\right)\end{array}$ & $\begin{array}{c}\text { MCSA of } 1 / 4 \\
\text { proximal region } \\
\left(\mathrm{cm}^{2}\right)\end{array}$ & & $\begin{array}{l}\text { MCSA of middle } \\
\text { region } \\
\left(\mathrm{cm}^{2}\right)\end{array}$ & $\begin{array}{c}\text { MCSA of } 1 / 4 \\
\text { distal region } \\
\left(\mathrm{cm}^{2}\right)\end{array}$ & & $\begin{array}{c}\text { MCSA of most } \\
\text { distal region } \\
\left(\mathrm{cm}^{2}\right)\end{array}$ \\
\hline & Neutral & $0.19 \pm 0.31$ & $10.48 \pm 2.84$ & & $20.27 \pm 4.48$ & $11.70 \pm 3.18$ & & $1.89 \pm 1.36$ \\
\hline \multirow[t]{3}{*}{ SOL } & Dorsal flexion & $0.35 \pm 0.37$ & $10.16 \pm 2.51$ & $* t$ & $20.08 \pm 4.89$ & $11.76 \pm 3.44$ & $* t$ & $2.13 \pm 1.51$ \\
\hline & Plantar flexion & $0.65 \pm 0.53$ & $12.32 \pm 3.46$ & & $20.54 \pm 5.00$ & $10.22 \pm 3.17$ & & $1.44 \pm 0.96$ \\
\hline & Neutral & $3.13 \pm 1.60$ & $9.05 \pm 2.02$ & & $12.71 \pm 3.58$ & $4.65 \pm 2.84$ & & $0.15 \pm 0.28$ \\
\hline \multirow[t]{3}{*}{ GM } & Dorsal flexion & $2.85 \pm 1.14$ & $8.73 \pm 1.82$ & & $12.59 \pm 3.36$ & $5.31 \pm 2.95$ & $* \dagger$ & $0.31 \pm 0.64$ \\
\hline & Plantar flexion & $2.98 \pm 1.37$ & $8.84 \pm 2.23$ & & $13.02 \pm 3.75$ & $4.03 \pm 2.51$ & & $0.02 \pm 0.09$ \\
\hline & Neutral & $1.18 \pm 0.37$ & $4.33 \pm 1.01$ & & $6.38 \pm 1.69$ & $4.11 \pm 1.95$ & & $0.44 \pm 0.46$ \\
\hline \multirow[t]{3}{*}{ GL } & Dorsal flexion & $1.04 \pm 0.52$ & $4.00 \pm 1.08$ & & $6.45 \pm 1.81$ & $4.31 \pm 1.84$ & $* t$ & $0.50 \pm 0.46$ \\
\hline & Plantar flexion & $0.94 \pm 0.52$ & $4.41 \pm 0.99$ & & $6.60 \pm 1.99$ & $2.93 \pm 1.74$ & & $0.07 \pm 0.18$ \\
\hline & Neutral & $0.16 \pm 0.16$ & $4.54 \pm 0.16$ & & $5.89 \pm 1.23$ & $1.85 \pm 0.54$ & & $0.14 \pm 0.11$ \\
\hline \multirow[t]{2}{*}{ TA } & Dorsal flexion & $0.15 \pm 0.19$ & $5.31 \pm 0.33$ & $* \dagger$ & $5.75 \pm 0.98$ & $1.65 \pm 0.59$ & $*$ & $0.19 \pm 0.18$ \\
\hline & Plantar flexion & $0.18 \pm 0.20$ & $4.64 \pm 0.23$ & & $5.69 \pm 1.16$ & $2.02 \pm 0.62$ & & $0.17 \pm 0.09$ \\
\hline
\end{tabular}

*Significant difference in MCSA between dorsal flexion and plantar flexion $(\mathrm{p}<0.05)$.

$\dagger$ Significant difference in MCSA between neutral and dorsal flexion $(\mathrm{p}<0.05)$.

†Significant difference in MCSA between neutral and plantar flexion $(\mathrm{p}<0.05)$.

SOL: soleus; GM: gastrocnemius medial head; GL: gastrocnemius lateral head; TA: anterior tibial tuberosity.

Table 2. Comparison of the maximum muscle cros-sectional area (MCSA) values for each muscle

\begin{tabular}{lcccc}
\hline & $\begin{array}{c}\text { MCSA of neutral } \\
\text { position }\left(\mathrm{cm}^{2}\right)\end{array}$ & $\begin{array}{c}\text { MCSA of dorsal } \\
\text { flexion position }\left(\mathrm{cm}^{2}\right)\end{array}$ & $\begin{array}{c}\text { MCSA of plantar } \\
\text { flexion position }\left(\mathrm{cm}^{2}\right)\end{array}$ \\
\hline $\mathrm{SOL}$ & $21.20 \pm 4.36$ & $21.04 \pm 4.39$ & $21.52 \pm 4.80$ & \\
$\mathrm{GM}$ & $13.14 \pm 3.57$ & $12.82 \pm 3.43$ & $13.40 \pm 3.60$ & $*+$ \\
$\mathrm{GL}$ & $6.91 \pm 1.64$ & $6.89 \pm 1.66$ & $7.42 \pm 1.72$ & $*+$ \\
$\mathrm{TA}$ & $7.28 \pm 1.11$ & $7.54 \pm 1.13$ & $7.24 \pm 1.10$ & $*+$ \\
\hline
\end{tabular}

*Significant difference in MCSA between dorsal flexion and plantar flexion $(\mathrm{p}<0.05)$. $\dagger$ Significant difference in MCSA between neutral and dorsal flexion $(\mathrm{p}<0.05)$. $\ddagger$ Significant difference in MCSA between neutral and plantar flexion $(\mathrm{p}<0.05)$. SOL: soleus; GM: gastrocnemius medial head; GL: gastrocnemius lateral head; TA: anterior tibial tuberosity.

\section{DISCUSSION}

Kumagai et al. ${ }^{10)}$ compared the muscle thickness in each part of the body between the decubitus and standing positions using ultrasonography, and reported a significantly thinner muscle in the decubitus position than in the standing position. As this difference is associated with muscle compression due to gravity and the ground reaction force, there is a possibility that the muscle mass is underestimated by measurement in the resting decubitus position. Therefore, data on changes in lower leg muscle morphology provide more accurate morphological information that accounts for the effects of gravity when stretching is performed in a sitting position.

In this study, when the neutral position was compared with the dorsal/plantar flexion positions, MCSA in the distal quarter of the SOL, GM, and GL muscles as ankle plantar flexors were greater in the lengthened position than in the shortened position. The MCSA in the proximal quarter of the SOL was greater in the shortened position than in the lengthened position. In contrast, the MCSA in the proximal quarter of the TA as an ankle dorsiflexor was smaller in the neutral position than in dorsiflexion. Based on these findings, it was suggested that both the ankle dorsiflexors and plantar flexors change in morphology with changes in ankle position. Differences in MCSA tended to occur in both the proximal and distal quarters, compared with the ends or middle regions of the muscles. In the lengthened position, the muscle belly moved distally, resulting in distal movement of regions with a large MCSA. In the shortened position, the muscle belly moved proximally, resulting in proximal movement of regions with a large MCSA, and differences tended to occur in a quarter of the muscle belly. In the middle region, which was closer to the largest muscle belly site in both the lengthened and shortened positions, there were slight differences among the ankle positions. Moreover, differences were minimal at the ends of the muscles, because of the 
extremely small MCSA values.

The results of comparison between the plantar and dorsal flexion positions were similar to those between the neutral and dorsal/plantar flexion positions. In the proximal region, the MCSA of the SOL was larger in plantar flexion than in dorsal flexion. In the distal region, the MCSAs of the SOL, GM, and GL were smaller in plantar flexion than in dorsal flexion. In contrast, the MCSA of TA was smaller in plantar flexion than in dorsal flexion in the proximal region, but larger in plantar flexion than in dorsal flexion in the distal region. The larger MCSA in the lengthened position in the distal region suggests that longitudinal muscle lengthening resulted in a general decrease in MCSA and distal movement, as well as longitudinal lengthening of the entire muscle belly. A comparison of ankle position indicated that the MCSA differed by $10 \%$ or more at sites where significant differences were noted. Kinugasa et al. ${ }^{11)}$ stated that the cross-sectional area of a muscle decreased during stretching to maintain muscle volume. In this study, the changes in MCSA were less marked than those reported by Kinugasa et al. This is presumably because stretching was performed with the knee flexed in the current study, so differences in knee position affected the extent to which the gastrocnemius stretched. Regarding the SOL, the maximum value did not differ between ankle positions, suggesting that the stretch load was applied to the entire muscle belly locally rather than uniformly. Even in the same muscle, the muscle fiber type ${ }^{12,13)}$, pennation angle ${ }^{14)}$, and tissue structures, such as the capillaries $^{15)}$, were reported to differ among sites along the longitudinal axis, resulting in longitudinal differences in the severity of muscle atrophy ${ }^{16)}$ and the effects of interventions ${ }^{17}$. Kimura et al. ${ }^{5)}$ noted more marked inhibitory effects of muscle stretching on the disused SOL in the proximal region, indicating that this region is more markedly affected by mechanical stimuli for structural reasons. In addition, Cè et al. ${ }^{18)}$ reported the presence of aponeuroses and connective tissue in the distal region of the lower leg muscles, and only slight morphological changes with muscle stretching. However, in the present study, the degree of longitudinal movement of each region of the same muscle was not evaluated, and such measurement and evaluation will be necessary in future research. Concerning the other limitations of this study, as the activities of the lower leg muscles were not evaluated, the degree of influence of muscle contraction was unclear. In addition, the muscle belly was within the measurement interval in some participants but not others, and the entire muscle could not be evaluated. Hedayatpour et al. ${ }^{19)}$ reported that non-uniform effects of exercise in the longitudinal direction of the skeletal muscle result in muscle output imbalance, which changes joint load distribution, increasing the risk of injury. When implementing an intervention to prevent muscle atrophy, the MCSA may need to be kept equal along its longitudinal axis. In a study involving rats, Miyachi et al. ${ }^{20)}$ reported that differences in more proximal atrophy due to inactivity decreased along the longitudinal axis of a muscle as a result of heat stress and stretching. The results of this study suggested that stretching causes differences in the load placed upon sites along the longitudinal axis of a muscle in humans.

For the prevention and inhibition of muscle atrophy and in interventions for muscle hypertrophy, regions along the longitudinal axis of the muscle should therefore be considered. Future intervention methods based on the morphological changes of a muscle along its longitudinal axis with adjustments in joint position are needed. In addition to the muscle morphology, other elements such as blood flow should be analyzed.

In conclusion, this study is the first to evaluate changes in lower leg muscle morphology with changes in ankle position when sitting using MRI. At the sitting position, the morphological changes of lower leg muscle according to change of ankle joint position is not uniform in the longitudinal direction of the muscle. Therefore, it is necessary to develop an intervention considering the morphological change of the muscle in the longitudinal direction of the muscle for prevention and inhibition of muscle atrophy.

\section{Funding}

This work was supported by JSPS KAKENHI Grant Number JP16K01500.

\section{Conflict of interest}

No potential conflicts of interest are disclosed.

\section{ACKNOWLEDGMENT}

The authors thank Ms. Haruka Ishizaki and Ms. Tomoko Kataya for their help with this study.

\section{REFERENCES}

1) Horak FB, Nashner LM: Central programming of postural movements: adaptation to altered support-surface configurations. J Neurophysiol, 1986, 55: 13691381. [Medline] [CrossRef]

2) Loram ID, Maganaris CN, Lakie M: Paradoxical muscle movement during postural control. Med Sci Sports Exerc, 2009, 41: 198-204. [Medline] [CrossRef]

3) Liu MQ, Anderson FC, Pandy MG, et al.: Muscles that support the body also modulate forward progression during walking. J Biomech, 2006, 39: 2623-2630. [Medline] [CrossRef]

4) Pandy MG, Andriacchi TP: Muscle and joint function in human locomotion. Annu Rev Biomed Eng, 2010, 12: 401-433. [Medline] [CrossRef] 
5) Kimura S, Inaoka PT, Yamazaki T: Influence of passive stretching on inhibition of disuse atrophy and hemodynamics of rat soleus muscle. J Jpn Phys Ther Assoc, 2012, 15: 9-14. [Medline] [CrossRef]

6) Oudeman J, Mazzoli V, Marra MA, et al.: A novel diffusion-tensor MRI approach for skeletal muscle fascicle length measurements. Physiol Rep, 2016, 4: 1-12. [Medline] [CrossRef]

7) Morse CI, Thom JM, Reeves ND, et al.: In vivo physiological cross-sectional area and specific force are reduced in the gastrocnemius of elderly men. J Appl Physiol 1985, 2005, 99: 1050-1055. [Medline] [CrossRef]

8) Lee HD, Finni T, Hodgson JA, et al.: Soleus aponeurosis strain distribution following chronic unloading in humans: an in vivo MR phase-contrast study. J Appl Physiol 1985, 2006, 100: 2004-2011. [Medline] [CrossRef]

9) Ohno N, Miyati T, Hiramatsu Y, et al.: Quantitation of venous blood flow in gravity MRI: a phantom study. Med Imaging Inf Sci, 2017, 34 : 141-143.

10) Kumagai K: The influence of posture on muscle thickness measurement using B-mode ultrasound. Nagasaki Int Univ Rev, 2011, 11: 1-7.

11) Kinugasa R, Hodgson JA, Edgerton VR, et al.: Asymmetric deformation of contracting human gastrocnemius muscle. J Appl Physiol 1985, 2012 , 112: 463-470. [Medline] [CrossRef]

12) Wang LC, Kernell D: Proximo-distal organization and fibre type regionalization in rat hindlimb muscles. J Muscle Res Cell Motil, 2000, 21: 587-598. [Medline] [CrossRef]

13) Punkt K, Mehlhorn H, Hilbig H: Region- and age-dependent variations of muscle fibre properties. Acta Histochem, 1998, 100: 37-58. [Medline] [CrossRef]

14) Lansdown DA, Ding Z, Wadington M, et al.: Quantitative diffusion tensor MRI-based fiber tracking of human skeletal muscle. J Appl Physiol 1985, 2007, 103: 673-681. [Medline] [CrossRef]

15) De Ruiter CJ, De Haan A, Sargeant AJ: Physiological characteristics of two extreme muscle compartments in gastrocnemius medialis of the anaesthetized rat. Acta Physiol Scand, 1995, 153: 313-324. [Medline] [CrossRef]

16) Sakuma K, Saitoh A, Katsuta S: Denervation-induced region-specific changes in fibre types in the soleus and plantaris muscles of rats. Acta Neuropathol, 1997, 93: 129-135. [Medline] [CrossRef]

17) Sakuma K, Yamaguchi A, Katsuta S: Are region-specific changes in fibre types attributable to nonuniform muscle hypertrophy by overloading? Eur J Appl Physiol Occup Physiol, 1995, 71: 499-504. [Medline] [CrossRef]

18) Cè E, Longo S, Rampichini S, et al.: Stretch-induced changes in tension generation process and stiffness are not accompanied by alterations in muscle architecture of the middle and distal portions of the two gastrocnemii. J Electromyogr Kinesiol, 2015, 25: 469-478. [Medline] [CrossRef]

19) Hedayatpour N, Falla D: Non-uniform muscle adaptations to eccentric exercise and the implications for training and sport. J Electromyogr Kinesiol, 2012, 22: 329-333. [Medline] [CrossRef]

20) Miyachi R, Yamazaki T: Effects of static interventions on disuse atrophy of the rat soleus muscle at different sites along its longitudinal axis. J Phys Ther Sci, 2015, 27: 2317-2321. [Medline] [CrossRef] 